\title{
[P-W-473] AN ENU MUTAGENESIS SCREEN FOR DOMINANT GENETIC MODIFIERS OF THROMBOSIS IN THE FACTOR 5 LEIDEN MOUSE
}

\section{R.J. Westrick, S.L. Manning, M.E. Winn, G.M. Stotz, E. Sanford, D. Ginsburg. Human Genetics; Howard Hughes Medical Institute; Internal Medicine; Life Sciences Institute, University of Michigan, Ann Arbor, United States}

Introduction: Factor V Leiden, (F5Q) is the most common inherited risk factor for venous thrombosis. However, a relatively small percentage of people carrying F5Q will develop venous thrombosis in their lifetimes. This variability results from complex interactions between the environment and unknown genetic factors (modifier genes). We previously identified a synthetically lethal interaction between mice inheriting two copies of F5Q (F5Q/Q) and partial Tfpi (Tfpi+/-) deficiency (F5Q/Q Tfpi+/-). Complete Tfpi deficiency in mice is embryonic lethal, whereas heterozygosity is compatible with normal survival. However, all mice inheriting F5Q/Q and Tfpi+/die of thrombosis shortly after birth.

Methods: The F5Q/Q Tfpi+/- lethal interaction was utilized as the basis for a sensitized ENU mutagenesis screen designed to uncover novel dominant suppressor mutations that alter the hemostatic balance. Male F5Q/Q mice were ENU mutagenized and bred to F5Q/+ Tfpi+/- double heterozygous females. Surviving offspring were analyzed to identify rescued mice with the F5Q/Q Tfpi+/- genotype.

Results: Analysis of $5600 \mathrm{G} 1$ offspring to date has identified 64 mice that survived to weaning. Of the 12 F5Q/Q Tfpi+/- mutants tested for heritability, five produced additional F5Q/Q Tfpi+/progeny, demonstrating that the associated thrombosis suppressor is heritable. The latter progeny have been genotyped with a panel of markers spanning the genome. For one of these mutant lines, this analysis has mapped the corresponding suppressor mutation to a small region on Chromosome 9, with fine mapping to identify the corresponding gene underway.

Conclusions: We have demonstrated the existence of novel dominant mutations that improve hemostatic balance leading to survival of F5Q/Q Tfpi+/- mice. Characterization of the genes may provide novel insight into the regulation of hemostasis, as well as identifying potential candidates for thrombosis modifiers in humans.

Westrick RJ, Manning SL, Winn ME, Stotz GM, Sanford E, Ginsburg D. AN ENU MUTAGENESIS SCREEN FOR DOMINANT GENETIC MODIFIERS OF THROMBOSIS IN THE FACTOR 5 LEIDEN MOUSE. $J$ Thromb Haemost 2007; 5 Supplement 2: P-W-473

Date: Wednesday, July 11, 2007

Session Info: Poster: Thrombophilia and familial thrombosis

Room: Exhibition Area 